\title{
Synthesis and Biological Evaluation of Cyclic Imides with Coumarins and Azacoumarins
}

\author{
R. Marulasiddaiah ${ }^{1}$, Rajesh G. Kalkhambkar ${ }^{2}$, Manohar V. Kulkarni ${ }^{{ }^{*}}$ \\ ${ }^{1}$ Department of Chemistry, Karnatak University, Dharwad, India \\ ${ }^{2}$ Department chemistry, Karnatak Science College, Dharwad, India \\ Email: *manohar274@gmail.com
}

Received August 1, 2012; revised September 3, 2012; accepted September 17, 2012

\begin{abstract}
A series of new N-substituted cyclic imides of coumarins and azacoumarins have been synthesized. All the compounds were screened for their antibacterial and antifungal activities. $\alpha$-Amylase enzyme inhibition activities of some $\mathrm{N}$-substituted phthalimide derivatives have been explored. All the compounds were characterized by IR, ${ }^{1} \mathrm{H}-\mathrm{NMR}$, and GC-MS.
\end{abstract}

Keywords: $\alpha$-Amylase Enzyme; Coumarin and 1-Azacoumarin

\section{Introduction}

Exploration of new chemical entities (NCE) for biological applications has become an important area of research in the field of drug discovery [1,2]. Heterocyclic frameworks are present in majority of the clinically accepted drug molecules. An important feature of heterocycles is their ability to sustain enormous structural diversity which is very much necessary to establish lead compounds for a variety of pharmacological activities [3]. Nitrogen heterocycles have been recognized as privileged structures to bind the receptor sites of macromolecules, leading to the perturbation of their metabolic functions which is a characteristic feature of biologically active molecules [4]. Cyclic imides constitute an important class of compounds possessing bis-amide linkages with common nitrogen. They have been useful building blocks in the synthesis of natural products [5] and other heterocycles [6] as well. Their ability to cross biological membranes in-vivo, due to their hydrophobicity is well established [7]. In view of the favorable pharmacokinetic properties, derivatives of cyclic imides have been found to be exhibiting wide ranging biological properties (Figure 1) [8-10]. Cyclic imides with a para-sulphonamide group have been potential anti tubercular agents [11], incorporation of heterocyclic moiety in the imide portion or linkage with nitrogen has resulted in molecular matrices with potential anti inflammatory [12] and anti tumor activities [13].

Bis-amide linkage have been employed as core structures in the design and synthesis of peptidomimetics

\footnotetext{
"Corresponding author.
}

which inhibit amino transferases [14,15]. In view of their biological importance, cyclic imides have been synthesized from diols [16], under microwave irradiation [17] and other routes [18]. Recent study demonstrated that chamomile plant extract suppresses the growth of human cancer cells and inhibits the pancreatic $\alpha$-amylase enzyme inhibitor, major components of $M$. chamomilla belongs to three different chemical classes: sesquiterpenes, coumarins, and flavonoids [19]. Trans-chalcone, a biphenolic core structure of flavonoids precursor exhibited $\alpha$-amylase inhibitory activity [20]. Survey of literature indicated that succinimide linked coumarins at the allylic position with respect to the C3 - C4 double bond have been employed in imaging of protein kinase activity in living cells [21]. In view of the diverse biological properties associated with coumarins and 1-aza coumarins (carbostyrils) [22], it was thought of considerable interest to synthesize cyclic imides linked to these moieties. In the light of these observations the present paper reports the synthesis of cyclic imides with general structure with three points of structural diversity in Figure 2.

$\alpha$-Amylase, a salivary or pancreatic enzyme plays an important role in early breakdown of starch into glucose and maltose. Modulation of $\alpha$-amylase activity affects the utilization of carbohydrates as an energy source and stronger is this modulation, more significant is the reduction in the breakdown of complex carbohydrates. Some of the $\mathrm{N}$-substituted phthalimide derivatives of coumarins and 1-azacoumarins have been subjected to $\alpha$-amylase enzyme inhibition activity. Retro synthetic analysis for the target compounds (Figure 2) indicates that crucial C-N bond forming step can be achieved using 


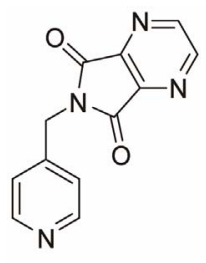<smiles>CN(C)c1ccc2c(CN3C(=O)CCC3=O)cc(=O)oc2c1</smiles><smiles>O=C1CCC(=O)N1Cc1cc(=O)oc2cc3c(cc12)OCO3</smiles>

Figure 1. Structurally related biologically active compounds.

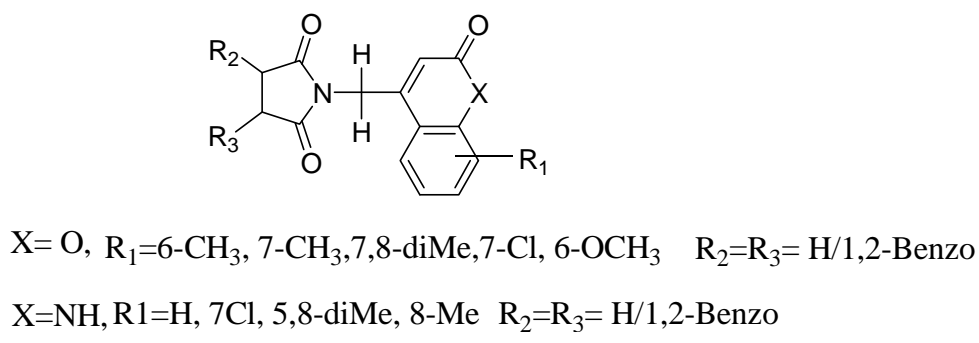

Figure 2. Cyclic imides with three points of structural diversity.

either aminomethyl compounds and the corresponding dicarboxylic acids/anhydrides by an amidation/ring insertion reaction or by using 4-halomethyl heterocycles and cyclic imides by a nucleophilic displacement at the allylic carbon. We have demonstrated the leaving group ability of 4-bromomethyl coumarins and 1-aza-4- bromomethyl coumarins using a variety of $\mathrm{O}, \mathrm{N}, \mathrm{S}$, nucleophiles leading to biologically active compounds [23-28]. In view of this we have employed the later route involving halomethyl heterocycles and cyclic imides.

\section{Materials and Methods}

Experimental: The melting points of the products were determined by open Capillary on Buchi apparatus. The IR spectra were recorded on a Nicole Impact-410 FT-I Spectrophotometer, using $\mathrm{KBr}$ pallets. ${ }^{1} \mathrm{HNMR}$ spectra were recorded on a Bruker-300 F $300 \mathrm{MHz}$ spectrometer in DMSO using TMS as an internal standard. Mass data were obtained from Shimadzu GC-MS. All the reagents were of analytical reagent grade and chemically pure. Solvents were distilled and dried before use.

Antibacterial activity: Agar diffusion method was employed [29] for inhibition activity against E. coli and Staphylococcus aureus bacteria, agar media was prepared by adding $10 \mathrm{~g}$ of peptone, $10 \mathrm{~g}$ of $\mathrm{NaCl}, 5 \mathrm{~g}$ of Yeast extract and $20 \mathrm{~g}$ of Agar into $1000 \mathrm{~mL}$ of distilled water. Initially the stock culture bacteria were revived and wells were made in the plate. Each plate was inoculated with $18 \mathrm{~h}$ old cultures and spread evenly on the plate. After 20 min, the wells were filled with compound at different concentrations. The control wells of Gentamycin were also prepared. All the plates were incubated at $37^{\circ} \mathrm{C}$ for $24 \mathrm{~h}$ and the diameter of inhibition zone was noted Table 1.

Antifungal Activity: Potato Dextrose Agar (PDA) media was employed [30] and was prepared by adding $250 \mathrm{~g}$ of boiled and peeled potato squeezed and filtered. To this filtrate $20 \mathrm{~g}$ of dextrose was added and the volume was made up to $1000 \mathrm{ml}$ by distilled water. Initially, the stock cultures of Aspergillus niger and Cladosporium were revived by inoculating in broth media grown at $27^{\circ} \mathrm{C}$ for $48 \mathrm{~h}$. The agar plates of the above media were prepared and wells were made in the plate. Each plate was inoculated with $18 \mathrm{~h}$ old cultures and spread evenly on the plate. After $20 \mathrm{~min}$, the wells were filled with compounds of different concentrations $(100 \mu \mathrm{g} / \mathrm{mL}, 200 \mu \mathrm{g} / \mathrm{mL}$ and 300 $\mu \mathrm{g} / \mathrm{mL}$ ). The control plates of Amphotericin were also prepared.All the plates were incubated at $27^{\circ} \mathrm{C}$ for $48 \mathrm{~h}$ and the diameter of inhibition zone was noted and shown

\section{in Table 2.}

$\alpha$-Amylase inhibitory activity: A stock sample concentration of $10 \mathrm{mg} / \mathrm{mL}$ concentration was prepared by using DMSO solvent. Reagent solution without test samples was used as control, activity of amylase was assayed with different concentrations of sample with control, DNS solution was prepared by dissolving $1 \mathrm{~g}$ of dinitro salicylic acid in $2 \mathrm{~N} \mathrm{NaOH}$ solution, $30 \mathrm{~g}$ of sodium potassium tartrate was added to the above solution and whole volume was diluted to $100 \mathrm{~mL}$. The test tubes with different concentrations of sample solutions were prepared and $1 \mathrm{ml}$ of sodium phosphate buffer $(50 \mathrm{mM}$. $\mathrm{pH}$ 7.0 - pH 7.3), $0.5 \mathrm{~mL}$ of starch, $0.1 \mathrm{~mL}$ of amylase enzyme(1 mg per $\mathrm{mL}$ sample in buffer) were added to each test tubes. The test tubes were incubated at $37^{\circ} \mathrm{C}$ for 10 min and $1 \mathrm{~mL}$ of DNS solution was added to each test tubes and the absorbance was measured at $540 \mathrm{~nm}$ against blank. Concentration of maltose liberated was determined by using standard maltose curve [31]. Enzyme activity was calculated and percentage of inhibition shown in the Table 3. 
Table 1. Anti-bacterial activity of N-substituted cyclic imide derivatives.

\begin{tabular}{|c|c|c|c|c|c|c|c|}
\hline \multirow[t]{2}{*}{ S. No } & \multirow[t]{2}{*}{ Compound } & \multicolumn{3}{|c|}{$\begin{array}{c}\text { E. coli (Gram - ve ) } \\
\text { Inhibition zones in cm (\%) }\end{array}$} & \multicolumn{3}{|c|}{$\begin{array}{l}\text { S. aureus (Gram + ve) } \\
\text { Inhibition zones in cm (\%) }\end{array}$} \\
\hline & & $300 \mu \mathrm{g}$ & $200 \mu \mathrm{g}$ & $100 \mu \mathrm{g}$ & $300 \mu \mathrm{g}$ & $200 \mu \mathrm{g}$ & $100 \mu \mathrm{g}$ \\
\hline 1 & $2 \mathrm{a}$ & 0.35 (13) & $0.15(7)$ & - & $0.40(17)$ & $0.26(12)$ & $0.21(12)$ \\
\hline 2 & $2 b$ & 0.65 (25) & $0.45(20)$ & 0.28 (13) & 0.67 (29) & $0.45(21)$ & - \\
\hline 3 & $2 c$ & $0.44(17)$ & $0.15(7)$ & - & $0.36(16)$ & $0.24(11)$ & - \\
\hline 4 & $2 d$ & $0.94(36)$ & $0.75(33)$ & $0.30(14)$ & $0.82(36)$ & 0.61 (29) & 0.35 (19) \\
\hline 5 & $2 \mathrm{e}$ & $1.20(46)$ & $0.88(38)$ & 0.35 (17) & $0.65(28)$ & $0.48(23)$ & $0.36(20)$ \\
\hline 6 & $2 f$ & $0.61(23)$ & $0.56(24)$ & $0.25(12)$ & $0.71(31)$ & $0.45(21)$ & - \\
\hline 7 & $2 \mathrm{~g}$ & $0.74(28)$ & 0.66 (29) & 0.35 (17) & $0.78(34)$ & $0.57(27)$ & $0.24(13)$ \\
\hline 8 & $2 \mathrm{~h}$ & 0.34 (13) & - & - & $0.27(12)$ & $0.21(10)$ & - \\
\hline 9 & $2 \mathrm{i}$ & $0.42(16)$ & $0.25(11)$ & - & $0.78(34)$ & $0.45(21)$ & 0.33 (18) \\
\hline 10 & $3 a$ & $0.51(20)$ & $0.32(14)$ & $0.20(10)$ & $0.50(22)$ & 0.35 (17) & - \\
\hline 11 & $3 b$ & $0.67(26)$ & $0.45(20)$ & $0.31(15)$ & $0.34(15)$ & $0.30(14)$ & $0.24(13)$ \\
\hline 12 & $3 c$ & 0.45 (17) & $0.22(10)$ & - & $0.41(18)$ & $0.25(12)$ & - \\
\hline 13 & $3 d$ & $1.44(55)$ & $0.95(41)$ & $0.53(25)$ & $1.30(57)$ & $0.95(45)$ & $0.54(30)$ \\
\hline 14 & 3e & 0.95 (37) & 0.65 (28) & 0.40 (19) & $1.11(48)$ & $0.88(42)$ & $0.51(28)$ \\
\hline 15 & $3 f$ & 0.95 (37) & $0.60(26)$ & 0.38 (18) & 0.92 ( 40) & $0.85(40)$ & 0.42 (23) \\
\hline 16 & $3 g$ & $1.13(43)$ & $0.74(32)$ & 0.61 (29) & $1.25(54)$ & $0.97(46)$ & $0.57(32)$ \\
\hline 17 & 3h & $0.30(12)$ & $0.15(7)$ & - & $0.46(20)$ & $0.30(14)$ & $0.22(12)$ \\
\hline 18 & $3 \mathrm{i}$ & $0.45(20)$ & 0.20 (9) & - & $0.71(31)$ & $0.41(20)$ & - \\
\hline 19 & Gentamycin & 2.6 & 2.3 & 2.1 & 2.3 & 2.1 & 1.8 \\
\hline
\end{tabular}

Control: DMSO.

Table 2. Anti-fungal activity of cylic imide derivatives.

\begin{tabular}{|c|c|c|c|c|c|c|c|}
\hline \multirow{2}{*}{ S. No } & \multirow{2}{*}{ Compound } & \multicolumn{3}{|c|}{$\begin{array}{c}\text { Asperigillus niger } \\
\text { Zone of Inhibition in } \mathrm{cm}(\%)\end{array}$} & \multicolumn{3}{|c|}{$\begin{array}{c}\text { Cladosporium } \\
\text { Zone of Inhibition in } \mathrm{cm}(\%)\end{array}$} \\
\hline & & $300 \mu g$ & $200 \mu g$ & $100 \mu g$ & $300 \mu g$ & $200 \mu g$ & $100 \mu g$ \\
\hline 1 & $2 \mathrm{a}$ & $0.21(16)$ & - & - & $0.15(14)$ & $0.10(10)$ & - \\
\hline 2 & $2 b$ & $0.28(22)$ & $0.20(21)$ & - & $0.22(20)$ & 0.18 (19) & - \\
\hline 3 & $2 c$ & $0.44(34)$ & 0.31 (33) & $0.19(24)$ & $0.18(16)$ & - & - \\
\hline 4 & $2 d$ & $0.57(44)$ & $0.40(43)$ & 0.23 (29) & $0.48(43)$ & $0.30(32)$ & 0.20 (29) \\
\hline 5 & $2 \mathrm{e}$ & $0.64(49)$ & $0.44(47)$ & $0.37(46)$ & 0.39 (35) & $0.22(23)$ & $0.14(20)$ \\
\hline 6 & $2 f$ & $0.30(23)$ & $0.13(14)$ & - & $0.25(23)$ & 0.15 (16) & - \\
\hline 7 & $2 g$ & $0.42(32)$ & $0.30(32)$ & $0.23(29)$ & $0.52(47)$ & 0.35 (37) & $0.22(31)$ \\
\hline 8 & $2 \mathrm{~h}$ & 0.35 (27) & $0.25(27)$ & - & $0.28(25)$ & 0.15 (16) & - \\
\hline 9 & $2 \mathrm{i}$ & 0.48 (37) & $0.30(32)$ & - & $0.35(32)$ & $0.16(17)$ & $0.10(14)$ \\
\hline 11 & $3 b$ & $0.36(28)$ & 0.27 (29) & $0.21(26)$ & $0.34(31)$ & $0.22(23)$ & $0.10(14)$ \\
\hline 12 & $3 c$ & $0.24(18)$ & $0.16(17)$ & - & $0.23(21)$ & $0.16(17)$ & - \\
\hline 13 & $3 d$ & $0.71(55)$ & $0.45(48)$ & $0.32(40)$ & $0.42(38)$ & 0.28 (29) & $0.15(21)$ \\
\hline 14 & 3e & $0.47(36)$ & 0.31 (33) & $0.25(31)$ & 0.38 (35) & $0.26(27)$ & $0.23(33)$ \\
\hline 15 & $3 f$ & $0.36(28)$ & $0.24(25)$ & 0.15 (18) & $0.36(33)$ & 0.18 (19) & $0.10(14)$ \\
\hline 16 & $3 g$ & 0.45 (35) & 0.27 (29) & $0.17(21)$ & $0.48(44)$ & $0.30(32)$ & $0.21(30)$ \\
\hline 17 & $3 \mathrm{~h}$ & $0.32(25)$ & $0.24(26)$ & - & $0.28(25)$ & 0.15 (16) & - \\
\hline 18 & $3 \mathrm{i}$ & $0.37(28)$ & - & - & $0.30(27)$ & $0.22(23)$ & $0.15(21)$ \\
\hline 19 & Amphotericin & 1.30 & 0.94 & 0.80 & 1.1 & 0.95 & 0.70 \\
\hline
\end{tabular}

Control: DMSO. 


\section{Results and Discussion}

A brief synthetic scheme employed during the present work for the title compounds in depicted in scheme 1 . The required 4-bromomethyl coumarins $1(\mathrm{X}=\mathrm{O})$ were prepared by Pechmann cyclization using various Phenols and 4-bromoethyl acetoacetate [32]. Reaction of succinimide using acetone and activated anhydrous potassium carbonate under reflux conditions resulted in cyclic imides 2a-e $(\mathrm{X}=\mathrm{O})$. Formation of compounds 2a-e was confirmed by ${ }^{1} \mathrm{H}$-NMR. The methylene protons in $\mathbf{1}$ $\left(\mathrm{X}=\mathrm{O}, \mathrm{R}=6-\mathrm{CH}_{3}\right)$ appeared as a singlet at $4.4 \mathrm{ppm}$ where as in cyclic imides $\mathbf{2 a}$, the methylene protons were deshielded and resonated at $5.0 \mathrm{ppm}$. The 1-aza-4-bromomethylcoumarin was prepared by the bromination of acetoacetanilide and cyclising the resulting $\omega$-bromoacetoacetanilide using sulphuric acid [33]. Under identical conditions cyclic imides $\mathbf{2 f}-\mathbf{i}(\mathrm{X}=\mathrm{NH})$ were obtained. Under these optimized conditions of a number of cyclic imides 2a-i were obtained.Finally the ORTEP diagram of the compound $\mathbf{2 c}(\mathrm{X}=\mathrm{O}, \mathrm{R}=7,8$-diMe) obtained by diffraction studies [34] has confirmed the structures of the products obtained in this reaction. The present methodology was extended for the reaction of phthalimide with various 4-bromomethyl coumarins and azacoumarins to obtain compounds 3a-i in good yields. The various Nsubstituted cyclic imides of coumarins and azacoumarins have been shown in Scheme 1.

\section{Antibacterial Activity}

Among the compounds 3a-i, 2-((7-chloro-2-oxo-2H-chromen-4-yl) methyl) isoindoline-1,3-dione (3d) has exhibited $41 \%$ inhibition and $55 \%$ inhibition against $\mathrm{E}$. at concentration of $200 \mu \mathrm{g} / \mathrm{mL}$ and $300 \mu \mathrm{g} / \mathrm{mL}$ respectively, It was also found to be active against $S$. aureus with inhibition of $45 \%$ and $57 \%$ at above concentration, other compounds have shown moderate activity against both bacteria. 2-((7-chloro-2-oxo-1,2-dihydroquinolin-4-yl) methyl) isoindoline-1,3-dione (3g) was found to be good antibacterial agent against $S$. aureus with inhibition activity of $46 \%$ and $54 \%$ at $200 \mu \mathrm{g} / \mathrm{mL}$ and $300 \mu \mathrm{g} / \mathrm{mL}$ concentrations, rest of the compounds exhibited moder- ate antibacterial activity. N-substituted succinimide derivatives of (2a-e) have not shown significant antibacterial activity except 1-((6-methoxy-2-oxo-2H-chromen4-yl) methyl) pyrrolidine-2,5-dione (2e). All other compounds of this series have shown moderate antibacterial activity.

\section{Antifungal Activity}

Among the series 2a-i both the compounds $\mathbf{2 d}$ and $\mathbf{2 e}$ exhibited significant inhibition activity against $A$. niger at $200 \mu \mathrm{g} / \mathrm{mL}$ and $300 \mu \mathrm{g} / \mathrm{mL}$.Compound $\mathbf{2 g}$ exhibited $47 \%$ inhibition against Cladosporium at $300 \mu \mathrm{g} / \mathrm{mL}$ concentration, rest of the compounds have shown moderate activity. Apart from 3a-i series, the compound 3d has shown remarkable inhibition against $A$. niger with $48 \%$ and $55 \%$ inhibition at $200 \mu \mathrm{g} / \mathrm{mL}$ and $300 \mu \mathrm{g} / \mathrm{mL}$ concentration respectively. All other compounds have exhibited moderate activity.

\section{1. $\alpha$-Amylase Inhibitory Activity}

Among the N-substituted phthalimide derivatives of azacoumarin compounds, $\mathbf{3 f}$ and $\mathbf{3 h}$ exhibited amylase enzyme inhibition activity at different concentration but $\mathbf{3 g}$ and $\mathbf{3 i}$ enacted as supporters of hydrolyzing activity of $\alpha$-amylase enzyme, therefore, the concentration of maltose was found to be more than that of the control. All the N-substituted phthalimides of coumarins were found to exhibit $\alpha$-amylase enzyme inhibitory activity except compound $3 \mathbf{e}$.

The relative enzyme inhibition activity of various $\mathrm{N}$ substituted phthalimides, at different concentrations has been shown in Figure 3. The decline in enzyme activity at different concentrations of $\mathbf{3 d}$ is compared with control and relative inhibition activity is shown in Figure 4.

Remarkable amylase inhibitory activity was observed by 2-((7-chloro-2-oxo-2H-chromen-4-yl)methyl) isoindoline-1,3-dione (3d) at $10 \mu \mathrm{g}$, suppressed the enzyme activity by $6.15 \%$, at $50 \mu \mathrm{g}$ by $10.99 \%$ and at $100 \mu \mathrm{g}$ the enzymatic activity was declined by $13.41 \%$. Various ions have also been shown to affect the activity of the<smiles>[X]c1ccccc1CN1C(=O)CCC1=O</smiles>

2a-i

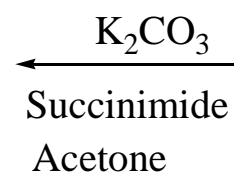

Acetone<smiles>[R]#Cc1ccccc1CBr</smiles>

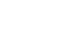

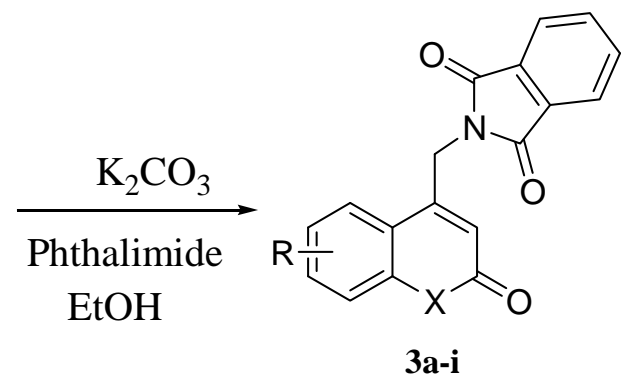

Scheme 1. Synthesis of $\mathrm{N}$-substituted cyclic imides 2a-i \& 3a-i. $\mathrm{X}=\mathrm{O}, \mathrm{R}=6-\mathrm{CH}_{3}, 7 \mathrm{CH}_{3}, 7,8-\mathrm{diMe}, 7-\mathrm{Cl}, \mathbf{6 - O M e}, \mathrm{X}=\mathrm{NH}, \mathrm{R}=$ H,7-Cl,5,8-diMe,8-Me. 
Table 3. $\alpha$-amylase inhibitory activity of some of the $\mathrm{N}$-substituted phthalimide derivatives.

\begin{tabular}{|c|c|c|c|c|c|}
\hline Sample & Quantity & OD at $540 \mathrm{~nm}$ & $\begin{array}{c}\text { Concentration of } \\
\text { Maltose liberated }(\mu \mathrm{g})\end{array}$ & $\begin{array}{c}\text { Activity } \\
(\mu \mathrm{moles} / \mathrm{ml} / \mathrm{min})\end{array}$ & $\%$ Activity \\
\hline \multirow{3}{*}{ 3a } & $100 \mu \mathrm{g}$ & 1.98 & 160 & 0.0441 & 97.10 \\
\hline & $200 \mu \mathrm{g}$ & 1.91 & 146 & 0.0405 & 89.01 \\
\hline & $500 \mu g$ & 1.74 & 139 & 0.0385 & 84.62 \\
\hline \multirow{3}{*}{$3 \mathrm{~b}$} & $100 \mu g$ & 1.98 & 159 & 0.0422 & 97.30 \\
\hline & $200 \mu \mathrm{g}$ & 1.89 & 153 & 0.0424 & 93.19 \\
\hline & $500 \mu \mathrm{g}$ & 1.79 & 143 & 0.0396 & 87.03 \\
\hline \multirow{3}{*}{ 3c } & $100 \mu \mathrm{g}$ & 1.99 & 160 & 0.0444 & 97.58 \\
\hline & $200 \mu \mathrm{g}$ & 1.92 & 166 & 0.0460 & 94.11 \\
\hline & $500 \mu g$ & 1.83 & 144 & 0.0399 & 87.69 \\
\hline \multirow{3}{*}{ 3e } & $100 \mu \mathrm{g}$ & 2.10 & 168 & 0.0466 & 102.42 \\
\hline & $200 \mu \mathrm{g}$ & 1.98 & 173 & 0.0480 & 105.49 \\
\hline & $500 \mu \mathrm{g}$ & 1.94 & 169 & 0.0469 & 103.08 \\
\hline \multirow{3}{*}{$3 f$} & $100 \mu g$ & 1.99 & 160 & 0.0444 & 97.58 \\
\hline & $200 \mu g$ & 1.92 & 154 & 0.0427 & 93.85 \\
\hline & $500 \mu \mathrm{g}$ & 1.89 & 151 & 0.0419 & 92.09 \\
\hline \multirow{3}{*}{$3 g$} & $100 \mu \mathrm{g}$ & 1.99 & 160 & 0.0444 & 97.58 \\
\hline & $200 \mu \mathrm{g}$ & 2.10 & 168 & 0.0466 & 102.42 \\
\hline & $500 \mu \mathrm{g}$ & 1.96 & 171 & 0.0474 & 104.18 \\
\hline \multirow{3}{*}{$3 \mathrm{~h}$} & $100 \mu \mathrm{g}$ & 1.94 & 157 & 0.0437 & 96.05 \\
\hline & $200 \mu \mathrm{g}$ & 1.90 & 152 & 0.0421 & 92.53 \\
\hline & $500 \mu \mathrm{g}$ & 1.86 & 149 & 0.0413 & 90.77 \\
\hline \multirow{3}{*}{$3 i$} & $100 \mu \mathrm{g}$ & 2.10 & 168 & 0.0466 & 102.42 \\
\hline & $200 \mu \mathrm{g}$ & 2.10 & 168 & 0.0466 & 102.42 \\
\hline & $500 \mu \mathrm{g}$ & 1.96 & 171 & 0.0474 & 104.18 \\
\hline \multirow{3}{*}{$3 d$} & $100 \mu \mathrm{g}$ & 1.81 & 142 & 0.0394 & 86.59 \\
\hline & $50 \mu \mathrm{g}$ & 1.86 & 146 & 0.0405 & 89.01 \\
\hline & $10 \mu \mathrm{g}$ & 1.91 & 154 & 0.0427 & 93.85 \\
\hline Control & - & 2.04 & 164 & 0.0455 & 100.00 \\
\hline
\end{tabular}

Solvent used: DMSO.

inhibitor. Lajolo et al. [35] reported the increase in the activity of the inhibitor against salivary amylase mediated by ions in the order Nitrate $>$ Chloride $>$ Bromide $>$ Iodide $>$ Thiocyanate. Gibbs and Alli reported that chloride ions are important for maximum activity while $\mathrm{Ca}$ ions increase the rate of initial binding of the inhibitor to the amylase. They also reported that $\mathrm{K}, \mathrm{Mg}$, sulfate and $\mathrm{Na}$ ions did not have any effects on the amylase inhibitor activity [36].

The starch-blocking mechanism of $\alpha$ amylase inhibitor: $\alpha$-amylase inhibitor action shows that the inhibitor is effective in preventing starch digestion by blocking access to the active site of the enzyme. During inhibition, several components of the inhibitor molecule, amylase molecule and the whole system have been reported to play important role in the mechanism. The chloride binding active sites of non-loop residues are Asp 197, Glu 233, Asp 300 and Arg 74 in amylase [35], This might be the reason that 2-((7-chloro-2-oxo- $2 \mathrm{H}$-chro- men-4-yl) methyl) isoindoline-1,3-dione (3d) was found to be the most competent as amylase enzyme inhibitor even at $10 \mu \mathrm{g} / \mathrm{mL}$ concentration.

General procedure for the preparation of compounds 2a-i and 3a-i: A mixture of substituted 4 bromomethyl coumarins/1-azacoumarins 1 (4 mmol), succinimide/phthalimide (4 mmol) and powdered anhydrous potassium carbonate $(4 \mathrm{mmol})$ in dry acetone/absolute alcohol $(25 \mathrm{~mL})$ was refluxed for $6 \mathrm{~h}$, completion of the reaction was monitored by TLC. After the completion of the reaction, the separated solid was filtered and washed with $10 \% \mathrm{HCl}$ and water, dried and recrystallized from suitable solvent.

1-((6-methyl-2-oxo-2H-chromen-4-yl)methyl)pyrrol idine-2, 5-dione (2a): White solid from acetic acid, yield $67 \%$, m.p. $185^{\circ} \mathrm{C}-187^{\circ} \mathrm{C}$. Anal. Calc. for $\mathrm{C}_{15} \mathrm{H}_{13} \mathrm{NO}_{4}$ (271.27): C, 66.41; H, 4.83; N, 5.16; Found: C, 66.29; H, 4.79; N, 5.10. IR $\left(v / \mathrm{cm}^{-1}\right)=1716 \mathrm{~cm}^{-1}(\mathrm{CO}) .{ }^{1} \mathrm{H}$ NMR $(\delta$ ppm) $2.38\left(s, 3 \mathrm{H}, \mathrm{C} 6-\mathrm{CH}_{3}\right), 2.79(s, 2 \mathrm{H}, \mathrm{C} 14-\mathrm{H}), 2.80(s$, 


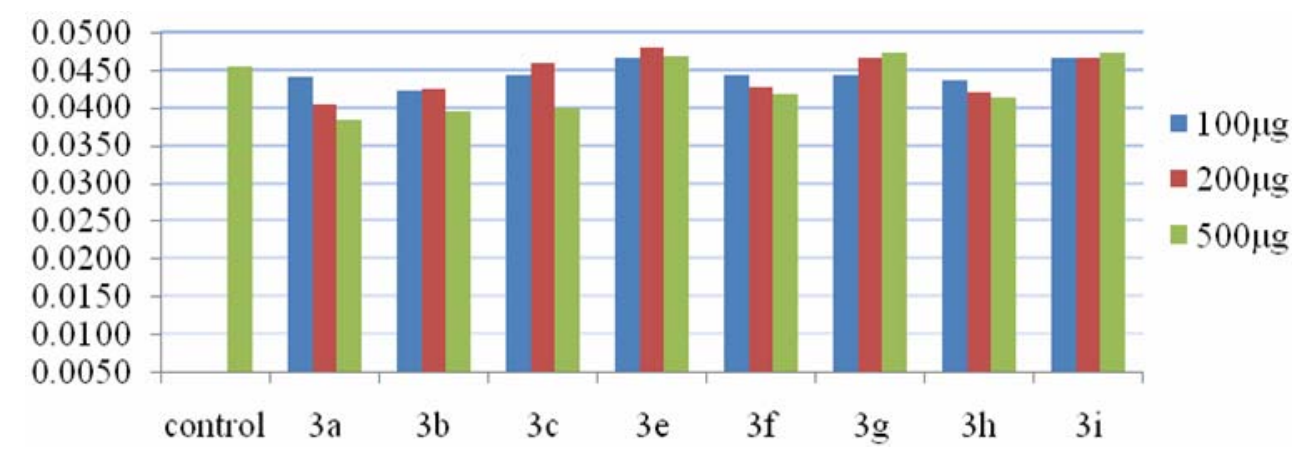

Figure 3. Graph showing the comparative analysis of samples with control.

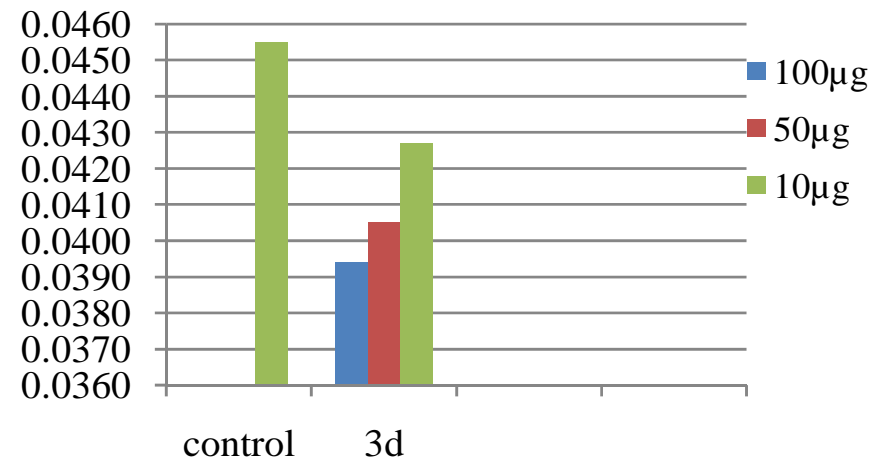

Figure 4. Graph showing the comparative activity of control and sample 3d.

2H, C15-6H), $4.88\left(s, 2 \mathrm{H}, \mathrm{C} 4-\mathrm{CH}_{2}\right), 6.32(s, 1 \mathrm{H}, \mathrm{C} 3-\mathrm{H})$, $7.27(d, 1 \mathrm{H}, \mathrm{C} 7-\mathrm{H}), 7.41(d, 1 \mathrm{H}, \mathrm{C} 8-\mathrm{H}), 7.56(s, 1 \mathrm{H}$, C5-H); GC-MS: m/z $271\left(\mathrm{M}^{+}, 100 \%\right)$.

1-((7-methyl-2-oxo-2H-chromen-4-yl)methyl)pyrrol idine-2,5-dione (2b): White solid from acetic acid, yield $72 \%$, m.p. $192^{\circ} \mathrm{C}-194^{\circ} \mathrm{C}$. Anal. Calc. for $\mathrm{C}_{15} \mathrm{H}_{13} \mathrm{NO}_{4}$ (271.27): C, 66.41; H, 4.83; N, 5.16; Found: C, 66.31; H, 4.80; N, 5.11. IR $\left(v / \mathrm{cm}^{-1}\right)=1716 \mathrm{~cm}^{-1}(\mathrm{CO}) .{ }^{1} \mathrm{H}$ NMR $(\delta$ ppm): 2.42 ( $\left.s, 3 \mathrm{H}, \mathrm{C} 7-\mathrm{CH}_{3}\right), 2.79\left(s, 2 \mathrm{H}, \mathrm{C} 14-\mathrm{H}, \mathrm{CH}_{2}\right)$, $2.80\left(s, 2 \mathrm{H}, \mathrm{C} 15-\mathrm{CH}_{2}\right), 4.78\left(s, 2 \mathrm{H}, \mathrm{C} 4-\mathrm{CH}_{2}\right), 6.34(s, 1 \mathrm{H}$, C3-H), 7.35 ( $s, 1 \mathrm{H}, \mathrm{C} 8-\mathrm{H}), 7.41(d, 1 \mathrm{H}, \mathrm{C} 6-\mathrm{H}), 7.71(d$, 1H, C5-H); GC-MS: m/z $271\left(\mathrm{M}^{+}, 100 \%\right)$.

1-((7,8-dimethyl-2-oxo-2H-chromen-4-yl)methyl)py rrolidine-2,5-dione (2c): White crystals from acetic acid yield $68 \%$, m.p. $212^{\circ} \mathrm{C}$. Anal. Calc. for $\mathrm{C}_{16} \mathrm{H}_{15} \mathrm{NO}_{4}$ (285.29): C, 67.36; H, 5.30; N, 4.91; Found: C, 67.28; H, 5.26; N, 4.89. IR $\left(v / \mathrm{cm}^{-1}\right)=1718 \mathrm{~cm}^{-1}(\mathrm{CO}) .{ }^{1} \mathrm{H}$ NMR $(\delta \mathrm{ppm})$ : $2.29\left(s, 3 \mathrm{H}, \mathrm{C} 7-\mathrm{CH}_{3}\right), 2.38\left(s, 3 \mathrm{H}, \mathrm{C} 8-\mathrm{CH}_{3}\right), 2.79(s, 2 \mathrm{H}$, C14-H), 2.80(s, 2H, C15-H), $4.75\left(s, 2 \mathrm{H}, \mathrm{C} 4-\mathrm{CH}_{2}\right), 6.24$ $(s, 1 \mathrm{H}, \mathrm{C} 3-\mathrm{H}), 7.25(d, 1 \mathrm{H}, \mathrm{C} 6-\mathrm{H}), 7.67(d, 1 \mathrm{H}, \mathrm{C} 5-\mathrm{H})$; GC-MS: m/z $285.29\left(\mathrm{M}^{+}, 100 \%\right)$.

1-((7-chloro-2-oxo-2H-chromen-4-yl)methyl)pyrroli dine-2,5-dione (2d): White solid from ethanol and DMF, yield 54\%, m.p. $223^{\circ} \mathrm{C}-224^{\circ} \mathrm{C}$. Anal. Calc. for $\mathrm{C}_{14} \mathrm{H}_{10} \mathrm{ClNO}_{4}$ (291.03): C, 57.65; H, 3.46; N, 4.80; Found: C, 57.39; H, $3.40 \mathrm{~N}, 4.74 . \operatorname{IR}\left(v / \mathrm{cm}^{-1}\right)=1718 \mathrm{~cm}^{-1},{ }^{1} \operatorname{HNMR}(\delta \mathrm{ppm})$ : $2.80\left(s, 2 \mathrm{H}, \mathrm{C} 14-\mathrm{CH}_{2}\right), 2.81\left(s, 2 \mathrm{H}, \mathrm{C} 15-\mathrm{CH}_{2}\right), 4.83(s$, $\left.2 \mathrm{H}, \mathrm{C} 4-\mathrm{CH}_{2}\right), 6.38(s, 1 \mathrm{H}, \mathrm{C} 3-\mathrm{H}), 7.30(s, 1 \mathrm{H}, \mathrm{C} 8-\mathrm{H})$,
7.48 (d, 1H, C6), $7.83(d, 1 \mathrm{H}, \mathrm{C} 5-\mathrm{H})$; GC-MS: $291\left(\mathrm{M}^{+}\right.$, $100 \%), 293\left(\mathrm{M}^{+} 2,28 \%\right)$.

1-((6-methoxy-2-oxo-2H-chromen-4-yl)methyl)pyrr olidine-2,5-dione (2e): Pale yellow solid from acetic acid, yield $67 \%$, m.p. $229^{\circ} \mathrm{C}-231^{\circ} \mathrm{C}$. Anal. Calc. for $\mathrm{C}_{19} \mathrm{H}_{13} \mathrm{NO}_{5}$ (287.27): C, 68.06; H, 3.91; N, 4.18; Found: C, 67.77; H, 3.86; N, 4.13. IR $\left(v / \mathrm{cm}^{-1}\right)=1718 \mathrm{~cm}^{-1}$ (CO), ${ }^{1} \mathrm{H}$ NMR $(\delta \mathrm{ppm}): 2.78\left(s, 2 \mathrm{H}, \mathrm{C} 14-\mathrm{CH}_{2}\right), 2.79(s$, $\left.2 \mathrm{H}, \mathrm{C} 15-\mathrm{CH}_{2}\right), 3.78\left(s, 3 \mathrm{H}, \mathrm{C} 6-\mathrm{OCH}_{3}\right), 6.26(s, 3 \mathrm{H}$, C3-H), 7.54 (d, 1H, C7-H), 7.69 ( $s, 1 \mathrm{H}, \mathrm{C} 5-\mathrm{H}), 7.81$ (d, 1H, C8-H). GC-MS: m/z $287\left(\mathrm{M}^{+}, 100 \%\right)$.

1-((1,2-dihydro-2-oxoquinolin-4-yl)methyl)pyrrolidi ne-2,5-dione (2f): White solid from acetic acid, yield $71 \%$, m.p. $216^{\circ} \mathrm{C}-218^{\circ} \mathrm{C}$. Anal. Calc for $\mathrm{C}_{14} \mathrm{H}_{12} \mathrm{~N}_{2} \mathrm{O}_{3}$ (255.26): C, 65.62; H, 4.72; N, 10.93; Found: C, 65.25; $\mathrm{H}, 4.67 ; \mathrm{N}, 10.89$. IR $\left(v / \mathrm{cm}^{-1}\right)=3438 \mathrm{~cm}^{-1}(\mathrm{NH}), 1706$ $\mathrm{cm}^{-1}$ (CO), $1663 \mathrm{~cm}^{-1}$ (lactam CO). ${ }^{1} \operatorname{HNMR}(\delta \mathrm{ppm})$ : $2.79\left(s, 4 \mathrm{H}\right.$, pyrrolidine- $\left.\mathrm{CH}_{2}\right), 4.77\left(s, 2 \mathrm{H}, \mathrm{C} 4-\mathrm{CH}_{2}\right), 6.24$ (s, 1H, C3-H), 7.23 - 7.87 (m, 4H, Ar-H), $11.89(s, 1 \mathrm{H}$, NH). GC-MS: m/z $256\left(\mathrm{M}^{+}, 100 \%\right)$.

1-((7-chloro-1,2-dihydro-2-oxoquinolin-4-yl)methyl )pyrrolidine-2,5-dione (2g): White solid from a ethanol+ dioxane, yield $67 \%$, m.p. $228^{\circ} \mathrm{C}-230^{\circ} \mathrm{C}$. Anal. Calc for $\mathrm{C}_{14} \mathrm{H}_{11} \mathrm{ClN}_{2} \mathrm{O}_{3}$ (290.05): C, 57.84; H, 3.81; N, 9.64; Found: C, 57.49; H, 3.76; N 9 .61. IR $\left(v / \mathrm{cm}^{-1}\right)=3353 \mathrm{~cm}^{-1}(\mathrm{NH})$, $1714 \mathrm{~cm}^{-1}$ (CO). $1667 \mathrm{~cm}^{-1}$ (lactam CO). ${ }^{1} \mathrm{HNMR}$ ( $\delta$ ppm): $2.80\left(s, 4 \mathrm{H}\right.$, pyrrolidine- $\left.\mathrm{CH}_{2}\right), 4.82\left(s, 2 \mathrm{H}, \mathrm{C} 4-\mathrm{CH}_{2}\right), 6.29$ (s, 1H, C3-H), $7.31(s, 1 \mathrm{H}, \mathrm{C} 8-\mathrm{H}), 7.45(d, 1 \mathrm{H}, \mathrm{C} 6-\mathrm{H})$, 
7.71 (d, 1H, C5-H), 11.91 ( $s, 1 \mathrm{H}, \mathrm{NH})$. GC-MS: m/z 290 $\left(\mathrm{M}^{+}, 100 \%\right) .292(\mathrm{M}+2,30 \%)$.

1,2-dihydro-5,8-dimethyl-2-oxoquinolin-4-yl)methy l)pyrrolidine-2,5-dione (2h): Pale green solid from acetic acid, yield $64 \%$, m.p. $234^{\circ} \mathrm{C}-236^{\circ} \mathrm{C}$. Anal. Calc for $\mathrm{C}_{16} \mathrm{H}_{16} \mathrm{~N}_{2} \mathrm{O}_{3}$ (284.31): C, 67.59; H, 5.67; N, 9.85; Found: C, 67.24; H, 5.61; N, 9.80; IR $\left(v / \mathrm{cm}^{-1}\right)=3338 \mathrm{~cm}^{-1}(\mathrm{NH})$, $1713 \mathrm{~cm}^{-1}$ (CO). $1665 \mathrm{~cm}^{-1},{ }^{1} \operatorname{HNMR}(\delta \mathrm{ppm}): 2.37$ (s, 3H, $\left.\mathrm{C} 8-\mathrm{CH}_{3}\right), 2.42\left(s, 3 \mathrm{H}, \mathrm{C} 5-\mathrm{CH}_{3}\right), 2.78(s, 4 \mathrm{H}$, pyrrolidine$\left.\mathrm{CH}_{2}\right)$, $4.73\left(s, 2 \mathrm{H}, \mathrm{C} 4-\mathrm{CH}_{2}\right), 6.27(s, 1 \mathrm{H}, \mathrm{C} 3-\mathrm{H}), 7.48(d$, 1H, C7-H), 7.65 ( $d, 1 \mathrm{H}, \mathrm{C} 6-\mathrm{H}), 11.78(s, 1 \mathrm{H}, \mathrm{NH})$. GC-MS: $\mathrm{m} / \mathrm{z} 270\left(\mathrm{M}^{+}, 100 \%\right)$.

1-((1,2-dihydro-8-methyl-2-oxoquinolin-4-yl)methyl) pyrrolidine-2,5-dione (2i): White solid from acetic acid, yield $58 \%$, m.p. $251^{\circ} \mathrm{C}-253^{\circ} \mathrm{C}$. Anal. Calc for $\mathrm{C}_{15} \mathrm{H}_{14} \mathrm{~N}_{2} \mathrm{O}_{3}$ (270.28): C, 66.66; H, 5.22; N, 10.36; Found: C, 66.29; $\mathrm{H}, 5.17 ; \mathrm{N}, 10.31$. IR $\left(v / \mathrm{cm}^{-1}\right)=3312 \mathrm{~cm}^{-1}(\mathrm{NH}), 1715$ $\mathrm{cm}^{-1}$ (CO), $1660 \mathrm{~cm}^{-1}$ (lactam CO). ${ }^{1} \mathrm{H}$ NMR ( $\left.\delta \mathrm{ppm}\right)$ : $2.37\left(s, 3 \mathrm{H}, \mathrm{C} 8-\mathrm{CH}_{3}\right), 2.78\left(s, 4 \mathrm{H}\right.$, pyrrolidine- $\left.\mathrm{CH}_{2}\right), 4.78$ $\left(s, 2 \mathrm{H}, \mathrm{C} 4-\mathrm{CH}_{2}\right), 6.32(s, 1 \mathrm{H}, \mathrm{C} 3-\mathrm{H}), 7.27(t, 1 \mathrm{H}, \mathrm{C} 6-\mathrm{H})$, $7.44(d, 1 \mathrm{H}, \mathrm{C} 7-\mathrm{H}), 7.66$ (d, 1H, C5-H), $11.87(s, 1 \mathrm{H}, \mathrm{NH})$. GC-MS: m/z $270\left(\mathrm{M}^{+}, 100 \%\right)$.

2-((6-methyl-2-oxo-2H-chromen-4-yl)methyl)isoind oline-1,3-dione (3a): White solid from acetic acid, yield $73 \%$, m.p. $263^{\circ} \mathrm{C}-265^{\circ} \mathrm{C}$. Anal. Calc. for $\mathrm{C}_{19} \mathrm{H}_{13} \mathrm{NO}_{4}$ (319.3): C, 71.47; H, 4.10; N, 4.39; Found: C, 71.16; H, 4.05; N, 4.33. IR $\left(v / \mathrm{cm}^{-1}\right)=1775 \mathrm{~cm}^{-1}$ (cyclic CO), $1716 \mathrm{~cm}^{-1}(\mathrm{C}=\mathrm{O}) .{ }^{1} \mathrm{HNMR}(\delta \mathrm{ppm}): 2.42\left(s, 3 \mathrm{H}, \mathrm{C} 6-\mathrm{CH}_{3}\right)$, $5.02\left(s, 2 \mathrm{H}, \mathrm{C} 4-\mathrm{CH}_{2}\right), 6.36(s, 1 \mathrm{H}, \mathrm{C} 3-\mathrm{H}), 7.37$ - $7.78(m$, 7H, Ar-H), GC-MS: m/z $271\left(\mathrm{M}^{+}, 100 \%\right)$.

2-((7-methyl-2-oxo-2H-chromen-4-yl)methyl)isoind oline-1,3-dione (3b): White solid from acetic acid, yield $78 \%$, m.p. $271^{\circ} \mathrm{C}-273^{\circ} \mathrm{C}$, Anal. Calc for $\mathrm{C}_{19} \mathrm{H}_{13} \mathrm{NO}_{4}$ (319.3): C, 71.47; H, 4.10; N, 4.39; Found: C, 71.18; H, 4.06; N, 4.33. IR $\left(v / \mathrm{cm}^{-1}\right)=1776 \mathrm{~cm}^{-1}$ (cyclic CO), 1716 $\mathrm{cm}^{-1}$ (CO). ${ }^{1} \mathrm{HNMR}(\delta \mathrm{ppm}): 2.44(s, 3 \mathrm{H}, \mathrm{CH}), 5.01(s$, 2H, C4-CH $\mathrm{CH}_{2}, 6.32$ (s, 2H, C3-H), 7.26 - 7.75 (m, 7H, Ar-H). GC-MS: m/z $319.3\left(\mathrm{M}^{+}, 100 \%\right)$.

2-((7,8-dimethyl-2-oxo-2H-chromen-4-yl)methyl)iso indoline-1,3-dione (3c): White solid from acetic acid, yield $68 \%$, m.p. $225^{\circ} \mathrm{C}-227^{\circ} \mathrm{C}$. Anal. Calc for $\mathrm{C}_{20} \mathrm{H}_{15} \mathrm{NO}_{4}$ (333.4): C, 72.06; H, 4.54; N, 4.20; Found: C, 71.74; H, 4.49; N, 4.17. IR $\left(v / \mathrm{cm}^{-1}\right)=1774 \mathrm{~cm}^{-1}$ (cyclic CO), 1718 $\mathrm{cm}^{-1}$ (CO). ${ }^{1} \mathrm{H}$ NMR ( $\left.\delta \mathrm{ppm}\right): 2.37\left(s, 3 \mathrm{H}, \mathrm{C} 7-\mathrm{CH}_{3}\right), 2.42$ $(s, 3 \mathrm{H}, \mathrm{C} 8-\mathrm{H}), 5.02\left(s, 2 \mathrm{H}, \mathrm{C} 4-\mathrm{CH}_{2}\right), 6.34(s, 1 \mathrm{H}, \mathrm{C} 3-\mathrm{H})$, 7.35 - 7.72 ( $m, 6 \mathrm{H}, \mathrm{Ar}-\mathrm{H})$. GC-MS: m/z 333 (M+, 30\%).

2-((7-chloro-2-oxo-2H-chromen-4-yl)methyl)isoindo line-1,3-dione (3d): White solid from ethanol + DMF, yield $71 \%$, m.p. $241^{\circ} \mathrm{C}-243^{\circ} \mathrm{C}$. Anal. Calc for $\mathrm{C}_{18} \mathrm{H}_{10} \mathrm{ClNO}_{4}$ (339.03): C, 63.64; H, 2.97; N, 4.12; Found: C, 63.31; H, 4.08; N, 4.09. IR $\left(v / \mathrm{cm}^{-1}\right)=1776 \mathrm{~cm}^{-1}$ (cyclic CO), 1718 $\mathrm{cm}^{-1}$ (CO). ${ }^{1} \mathrm{HNMR}(\delta \mathrm{ppm}): 4.98\left(s, 2 \mathrm{H}, \mathrm{C} 4-\mathrm{CH}_{2}\right), 6.34$ $(s, 1 \mathrm{H}, \mathrm{C} 3-\mathrm{H}), 7.27$ - $7.76(m, 7 \mathrm{H}, \mathrm{Ar}-\mathrm{H}) . \mathrm{GC}-\mathrm{MS}: \mathrm{m} / \mathrm{z}$ $339\left(\mathrm{M}^{+}, 100 \%\right), 341(\mathrm{M}+2,29 \%)$.
2-((6-methoxy-2-oxo-2H-chromen-4-yl)methyl)isoin doline-1,3-dione (3e): Pale yellow solid from Acetic acid, yield $76 \%$, m.p. $210^{\circ} \mathrm{C}-212^{\circ} \mathrm{C}$. Anal. Calc for $\mathrm{C}_{19} \mathrm{H}_{13} \mathrm{NO}_{5}$ (335.30): C, 68.06; H, 3.91; N, 4.18; Found: C, 67.72; H, 3.88; N, 4.14. IR $\left(v / \mathrm{cm}^{-1}\right)=1774 \mathrm{~cm}^{-1}$ (cyclic CO), 1719 $\mathrm{cm}^{-1}$ (CO). ${ }^{1} \mathrm{HNMR}(\delta \mathrm{ppm}): 3.79\left(s, 3 \mathrm{H}, \mathrm{C} 6-\mathrm{OCH}_{3}\right)$, $5.02\left(s, 2 \mathrm{H}, \mathrm{C} 4-\mathrm{CH}_{2}\right), 6.36(s, 1 \mathrm{H}, \mathrm{C} 3-\mathrm{H}), 7.28-7.73(m$, 7H, Ar-H). GC-MS: m/z 335 (M+, 100\%).

2-((1,2-dihydro-2-oxoquinolin-4-yl)methyl)isoindoli ne-1,3-dione (3f): White solid from acetic acid, yield 74\%, m.p. $287^{\circ} \mathrm{C}-289^{\circ} \mathrm{C}$. Anal. Calc for $\mathrm{C}_{18} \mathrm{H}_{12} \mathrm{~N}_{2} \mathrm{O}_{3}$ (304.08): C, 71.05; H, 3.97; N, 9.21; Found: C, 70.78; H, 3.92; N, 9.16. IR $\left(v / \mathrm{cm}^{-1}\right)=3315 \mathrm{~cm}^{-1}(\mathrm{NH}), 1776 \mathrm{~cm}^{-1}$ (cyclic CO), $1716 \mathrm{~cm}^{-1}$ (CO), $1655 \mathrm{~cm}^{-1}$ (lactam CO). ${ }^{1} \mathrm{H}$ NMR ( $\left.\delta \mathrm{ppm}\right)$ : $4.85\left(s, 2 \mathrm{H}, \mathrm{CH}_{2}\right), 6.41(s, 1 \mathrm{H}, \mathrm{C} 3-\mathrm{H}), 7.34-7.87(m, 8 \mathrm{H}$, Ar-H), 11.88 ( $s, 1 \mathrm{H}, \mathrm{NH})$. GC-MS: m/z $304\left(\mathrm{M}^{+}, 100 \%\right)$.

2-((7-chloro-1,2-dihydro-2-oxoquinolin-4-yl)methyl) isoindoline-1,3-dione (3g): White solid from acetic acid, yield $65 \%$, m.p. $276^{\circ} \mathrm{C}-278^{\circ} \mathrm{C}$. Anal. Calc for $\mathrm{C}_{18} \mathrm{H}_{11} \mathrm{ClN}_{2} \mathrm{O}_{3}$ (338.05): C, 63.82; H, 3.27; N, 8.27; Found: C, 63.47; H, 3.23, N, 8.22. IR $\left(v / \mathrm{cm}^{-1}\right)=3355 \mathrm{~cm}^{-1}(\mathrm{NH})$, $1774 \mathrm{~cm}^{-1}$ (cyclic CO) $1718 \mathrm{~cm}^{-1}, 1663 \mathrm{~cm}^{-1}$ (CO), ${ }^{1} \mathrm{H}$ NMR ( $\delta$ ppm): $4.92\left(s, 2 \mathrm{H}, \mathrm{CH}_{2}\right), 6.31(s, 1 \mathrm{H}, \mathrm{C} 3-\mathrm{H})$, 7.39 - $7.88(m, 7 \mathrm{H}, \mathrm{Ar}-\mathrm{H}), 11.91(s, 1 \mathrm{H}, \mathrm{NH})$. GC-MS: $\mathrm{m} / \mathrm{z} 338\left(\mathrm{M}^{+}, 100 \%\right), 340(\mathrm{M}+2,28 \%)$.

2-((1,2-dihydro-5,8-dimethyl-2-oxoquinolin-4-yl)me thyl)isoindoline-1,3-dione (3h): White solid from acetic acid, yield $74 \%$, m.p. $291^{\circ} \mathrm{C}-293^{\circ} \mathrm{C}$. Anal. Calc for $\mathrm{C}_{20} \mathrm{H}_{16} \mathrm{~N}_{2} \mathrm{O}_{3}$ (332.12): C, 72.28; H, 4.85; N, 8.43; Found: C, 71.93; H, 4.79; N, 8.40. IR $\left(v / \mathrm{cm}^{-1}\right)=3291 \mathrm{~cm}^{-1}(\mathrm{NH})$, $1775 \mathrm{~cm}^{-1}$ (cyclic CO) $1718 \mathrm{~cm}^{1}, 1661 \mathrm{~cm}^{1}$ (CO). ${ }^{1} \mathrm{HNMR}$ $(\delta \mathrm{ppm}): 2.37\left(s, 3 \mathrm{H}, \mathrm{C} 8-\mathrm{CH}_{3}\right), 4.42\left(s, 3 \mathrm{H}, \mathrm{C} 5-\mathrm{CH}_{3}\right)$, $4.88\left(s, 2 \mathrm{H}, \mathrm{CH}_{2}\right), 6.45(s, 1 \mathrm{H}, \mathrm{C} 3 \mathrm{H}), 7.33-7.78(m, 6 \mathrm{H}$, ArH), 11.86 ( $s, 1 \mathrm{H}, \mathrm{NH})$. GC-MS: m/z $332\left(\mathrm{M}^{+}, 36 \%\right)$.

2-((1,2-dihydro-8-methyl-2-oxoquinolin-4-yl)methyl) isoindoline-1,3-dione (3i): White solid from ethanol + dioxane, yield $68 \%$, m.p. $279^{\circ} \mathrm{C}-281^{\circ} \mathrm{C}$. Anal. Calc for $\mathrm{C}_{19} \mathrm{H}_{14} \mathrm{~N}_{2} \mathrm{O}_{3}$ (318.10): C, 71.69; H, 4.43; N, 8.80; Found: C, 71.27; H, 4.40; N, 8.75. IR $\left(v / \mathrm{cm}^{-1}\right)=3310 \mathrm{~cm}^{-1}(\mathrm{NH})$, $1776 \mathrm{~cm}^{-1}$ (cyclic CO) $1715 \mathrm{~cm}^{-1}, 1659 \mathrm{~cm}^{1}$ (CO), ${ }^{1} \mathrm{HNMR}$ ( $\delta$ ppm): $2.44\left(s, 3 \mathrm{H}, \mathrm{C} 8-\mathrm{CH}_{3}\right), 4.86\left(s, 2 \mathrm{H}, \mathrm{CH}_{2}\right), 6.45$ $(s, 1 \mathrm{H}, \mathrm{C} 3 \mathrm{H}), 7.327 .78(m, 6 \mathrm{H}, \mathrm{ArH}), 11.87(s, 1 \mathrm{H}, \mathrm{NH})$. GC-MS: m/z $318\left(\mathrm{M}^{+}, 100 \%\right)$.

\section{Conclusion}

The present study has shown that antimicrobial activity of $\mathrm{N}$-substituted phthalimide derivatives of coumarins and 1-azacoumarins are relatively more than N-substituted succinimide derivatives the introduction of methyl groups into the coumarin and 1-azacoumarin have declined their antimicrobial activity whereas the chloro and methoxy substituted coumarin and 1-azacoumarins have enhanced their antimicrobial activity. The substituted 
phthalimide derivatives of coumarins have shown amylase enzyme inhibition activity except ((6-methoxy-2-oxo -2H-chromen-4-yl)methyl) isoindoline-1,3-dione 3e, but some 1-azacoumarin cyclic imides exhibited enzyme supporting activity and enhanced the hydrolyzing ability of amylase enzyme. The compound 2-((7-chloro-2-oxo-2Hchromen-4-yl)methyl) isoindoline-1,3-dione $3 \mathbf{d}$ was found to be most efficient amylase inhibitor expected to be active in controlling hyperglycemia and useful in the management of type II diabetes and this compound could be used to design effective inhibitors of alpha-amylase. Simple synthetic method can be employed to synthesis wide range of cyclic imide derivatives by inexpensive readily available ecofriendly reagents.

\section{REFERENCES}

[1] C. Lipinski and A. Hopkins. "Navigating Chemical Space for Biology and Medicine,” Nature, Vol. 432, 2004, pp. 855-861. doi:10.1038/nature03193

[2] S. L. Schreiber, "Target-Oriented and Diversity-Oriented Organic Synthesis in Drug Discovery,” Science, Vol. 287, No. 5460, 2000, pp. 1964-1969. doi:10.1126/science.287.5460.1964

[3] R. Dua, S. Shrivastava, S. K. Sonawane and S. K. Srivastava, "Pharmacological Significance of Synthetic Heterocycles Scaffold: A Review," Advances in Biological Research, Vol. 5, No. 3, 2011, pp. 120-144.

[4] P. M. Chauhan and S. K. Srivastava, "Recent Developments in the Combinatorial Synthesis of Nitrogen Heterocycles Using Solid Phase Technology," Combinatorial Chemistry \& High Throughput Screening, Vol. 4, No. 1, 2001, pp. 35-51.

[5] B. Teng, J. Zheng, H. Huang and P. Huang, "Enantioselective Synthesis of Glutarimide Alkaloids," Chinese Journal of Chemistry, Vol. 29, No. 7, 2011, pp. 13121318. doi:10.1002/cjoc.201180248

[6] A. F. M. Fahmy, "Heterocycles as Versatile Building Blocks in Different Synthetic Strategies," Archive for Organic Chemistry, Vol. 7, 2006, pp. 395-415.

[7] M. K. Haergreeves, J. G. Prtichard and H. R. Dav, “Cyclic Carboxylic Monoimides,” Chemical Reviews, Vol. 70, No. 4, 1970, pp. 439-469. doi:10.1021/cr60266a001

[8] V. C. Filho, R. Correa, Z. Vaz, J. B. Calixto, R. J. Nunes, A. D. Andricopulo and R. A. Yunes, "Further Studies on Analgesic Activity of Cyclic Imides II,” Farmaco, Vol. 53, No. 1,1998, pp. 55-57. doi:10.1016/S0014-827X(97)00006-2

[9] D. E. Butler, J. D. Leonard, B. W. Caprathe, Y. J. Litalien, M. R. Pavia, F. M. Hershenson, P. H. Poschel and J. G. Marriott, "Amnesia-Reversal Activity of a Series of Cyclic Imides,” Journal of Medicinal chemistry, Vol. 30, No. 3, 1987, pp. 498-503.

[10] A. D. Andricopulo, L. A. Muller, V. C. Filho, G. S. Cani, J. F. Roos, A. R. Santos, R. J. Nunes, R. A. Yunes and Farmaco, "Analgesic Activity of Cyclic Imides: 1,8Naphthalimide and 1,4,5,8-Naphthalenediimide Deriva- tives,” Farmaco, Vol. 55, No. 4, 2000, pp. 319-321. doi:10.1016/S0014-827X(00)00027-6

[11] K. N. de Oliveira, L. D. Chiaradia, P. G. A. Martins, A. Mascarello, M. N. S.Cordeiro, R.V. C. Guido, A. D. Andricopulo, R. A. Yunes, R. J. Nunes, J. Vernalb and H. Terenzi, "Sulfonyl-Hydrazones of Cyclic Imides Derivatives as Potent Inhibitors of the Mycobacterium tuberculosis Protein Tyrosine Phosphatase B (PtpB),” Medicinal Chemistry Communication, Vol. 2, No. 3, 2011, pp. 500504.

[12] S. M. Sondhi, R. Rani, A. D. Dwiwedi and P. Roy, "Synthesis of Some Heterocyclic Imides and Azomethine Derivatives under Solvent Free Condition and Their AntiInflammatory Activity Evaluation,” Journal of Heterocyclic Chemistry, Vol. 46, No. 6, 2009, pp. 1369-1374. doi:10.1002/jhet.249

[13] S. M. Sondhi, R. Rani, P. Roy, S. K. Agrawal and A. K. Saxena, "ChemInform Abstract: Microwave-Assisted Synthesis of N-Substituted Cyclic Imides and Their Evaluation for Anticancer and Anti-inflammatory Activities," Bioorganic Medicinal Chemistry Letters, Vol. 19, No. 7, 2009, pp. 1534-1538. doi:10.1016/j.bmcl.2008.07.048

[14] Q. Li, H.Fang, X. Wang and W. Xu, "Novel CyclicImides Peptidomimetics as Amino Peptidase N Inhibitors," European Journal of Medicinal Chemistry, Vol. 45, No. 4, 2010, pp. 1618-1626. doi:10.1016/j.ejmech.2009.12.071

[15] Q. Li, H. Fang, X. Wang, L. Hu and W. Xu, "Novel Cyclic-Imide Peptidomimetics as Amino Peptidase N Inhibitors. Design, Chemistry and Activity Evaluation. Part I,” European Journal of Medicinal Chemistry, Vol. 44, No. 12, 2009, pp. 4815-4825. doi:10.1016/j.ejmech.2009.07.022

[16] J. Zhang, M. Senthilkumar, S. C. Ghosh and S. H. Hong, "Synthesis of Cyclic Imides from Simple Diols," Angewandte Chemie International Edition, Vol. 49, No. 36, 2010, pp. 6391-6395. doi:10.1002/anie.201002136

[17] Y. M. Hiji and B. Ellis, "Efficient Microwave Assisted Syntheses of Unsubstituted Cyclic Imides,” Heterocycles, Vol. 68, No. 11, 2006, pp. 2259-2267. doi:10.3987/COM-06-10841

[18] E. Benjamin and Y. Hijji, "The Synthesis of Unsubstituted Cyclic Imides Using Hydroxylamine under Microwave Irradiation,” Molecules, Vol. 13, No. 1, 2008, pp. 157-169.

[19] A. Kato and Y. Minoshima, "Protective Effects of Dietary Chamomile Tea on Diabetic Complications," Journal of Agricultural Food Chemistry, Vol. 56, No. 17, 2008, pp. 8206-8211. doi:10.1021/jf8014365

[20] M. Najafian, A. Ebrahim-Habibi, N. Hezareh, P. Yaghmaei, K. Parivar and B. Larijani, "Trans-Chalcone: A Novel Small Molecule Inhibitor of Mammalian AlphaAmylase,” Molecular Biology Reports, Vol. 38, No. 3, 2010, pp. 1617-1620.

[21] M. V. Kulkarni, G. M. Kulkarni, C.-H. Lin and C.-M. Sun, "Recent Advances in Coumarins and 1-Aza Coumarins as Versatile Biodynamic Agents," Current Medicinal Chemistry, Vol. 13, No. 23, 2006, pp. 2795-2889. 
doi:10.2174/092986706778521968

[22] H. Higashi, K. Sato, A. Ohtake, A. Omori, S. Yoshida and Y. Kudo, "Imaging of cAMP-Dependent Protein Kinase Activity in Living Neural Cells Using a Novel Fluorescent Substrate,” FEBS Letters, Vol. 414, No. 7, 1997, pp. 55-60. doi:10.1016/S0014-5793(97)00970-8

[23] M. D Ghate, M. V. Kulkarni, R. Shobha and S. Y. Kattimani, "Synthesis of Vanillin Ethers from 4-(Bromomethyl) Coumarins as Anti-Inflammatory Agents,” European Journal of Medicinal Chemistry, Vol. 38, No. 3, 2003, pp. 297302. doi:10.1016/S0223-5234(03)00016-3

[24] L. A. Shastri, K. Shivashankar and M. V. Kulkarni, "Facile Synthesis of Some Novel 4-\{3-Aryl-3,4-Dihydro$2 H$-Benzo[b][1,4] Thiazin-2-yl\}-2H-Chromen-2-One Derivatives," Journal of Sulfur Chemistry, Vol. 28, No. 6, 2007, pp. 625-630. doi:10.1080/17415990701591286

[25] G. M. Kulkarni, M. V. Kulkarni and V. D. Patil, "Heterocycles with Bridgehead Nitrogen: Synthesis, High Resolution NMR and Mass Spectra of 5 Aryloxymethyltetrazolo[1,5-a]Quinolines,” Journal of Indian Chemical Society, Vol. 74,1997, pp. 502-503.

[26] R. G. Kalkhambkara, G. M. Kulkarni, et al., "Synthetic and Biological Studies on Mono- and Bis-MethyleneBridged Heterocyclic Sulfides and Sulfones of Carbostyrils," Journal of Sulfur Chemistry, Vol. 30, No. 6, 2009, pp. 596-610. doi:10.1080/17415990903191745

[27] R. G. Kalkhambkar, G. M. Kulkarni and C. M. Kamanavalli, "Synthesis and Biological Activities of Some New Fluorinated Coumarins and 1-Aza Coumarins," European Journal of Medicinal Chemistry, Vol. 43, No. 10, 2008, pp. 2178-2188. doi:10.1016/j.ejmech.2007.08.007

[28] M. V. Kulkarni and V. D. Patil, "Synthesis and Biological Properties of Some 3-Heterocyclic Coumarins," Archiv der Pharmazie (Weinheim), Vo1. 314, No. 5, 1981, 435-
439.

[29] E. J. Threlfall, I. S. T. Fisher, l. R. Ward, H. Tschäpe and P. Gerner-Smidt, "Harmonization of Antibiotic Susceptibility Testing for Salmonella,” Microbial Drug Resistance, Vol. 5, No. 3, 1999, pp. 195-199. doi:10.1089/mdr.1999.5.195

[30] W. B. Hugo, A. B. Russel, S. P. Denyer, N. A. Hodges and S. P. German, "Pharmaceutical Microbiology," Blackwell Scientific Publication, 1987, pp. 187-202.

[31] S. Sadasivam and A. Manickam, "Biochemical Methods," New Age International (P) Limited, New Delhi, Vol. 2. 1996, pp. 124-126.

[32] M. V. Kulkarni, B. G. Pujar and V. D. Patil, "Studies on Coumarins II," Archiv der Pharmazie (Weinheim), Vol. 316, No. 1, 1983, pp. 15-17. doi:10.1002/ardp.19833160106

[33] M. Hasegawa, "Synthesis of 4-Bromomethyl Carbostyrils,” Pharmaceutical Bulletin Japan, Vol. 1, 1953, pp. 1-3.

[34] R. Marulasiddaiah, M. V. Kulkarni, D. Sharma and V. K. Gupta, "Crystal Structure of 1-(7,8-Dimethyl-2-oxo-2HChromen-4-ylmethyl)-PYRROLIDINE-2,5-Dione,” X-Ray Structure Analysis Online, Vol. 27, 2011, pp. 41-42. doi:10.2116/xraystruct.27.41

[35] F. M. Lajolo and F. F. Filho, "Partial Characterization of the Amylase Inhibitor of Black Beans (Phaseolus Vulgaris) Variety Rico 23," Journal of Agricultural Food Chemistry, Vol. 33, No. 1, 1985, pp. 132-138. doi:10.1021/jf00061a038

[36] B. Gibbs and I. Alli, "Characterization of a Purified $\alpha$-Amylase Inhibitor from White Kidney Beans (Phaseolus Vulgaris)," Food Research International, Vol. 31, No. 3, 1998, pp. 217-225. doi:10.1016/S0963-9969(98)00074-X 